\title{
A Research on Information System Applied to Optimizing Tourism Transport Issues
}

\author{
Huijuan Jin \\ Tourism and Air Service College, Guizhou Minzu University,China. \\ Email:47853433@qq.com
}

Keywords: Tourism transportation, information system, optimization

\begin{abstract}
In the system design based on the principles of combining the existing data to construct the tourism transport, by which the planning management information system frame and function modules of the system could be detailed designed, tourism transport related to information system development and application can improve the optimization. To solve the data analysis, management, spatial expression, spatial data visualization problems, provides a good platform for the planning and management departments, the relevant departments to promote the rational planning of resources in the system of optimizing tourism transport issue and layout, which can make due contributions to optimize tourism transport with information system.
\end{abstract}

\section{Introduction}

Analysis of regional planning information system as a decision-making tool in the planning department, analyze the regional achievements management planning and regional development space, plays an irreplaceable role. Tourism transportation is an important issue related to information system which can improve a site by including value-added features such as weather conditions, prices selected, updated exchange rates, destination issues links and other information things. In particular attention should be given to online tourism guest comments or survey forms for the demand of tourism transportation, result in adapting marketing mix variables to the information system related to tourism transportation ${ }^{[1]}$. They are interdependent and mutually reinforcing, which made the continuous development of statistical information related to tourism development. Tourist traffic is that tourists achieve to move from one location to another location to reach the space transfer process, from the task to solve tourists, from the tourist attractions, as well as from various regions to provide direct or indirect transport services by the use of certain statistical information. The products of statistical information related to tourism transportation, as well as transportation service cannot be stored, nor can they are divorced from production and consumption processes exist independently. But they can only be consumed in the production at the same time. Therefore, tourism transportation infrastructure must have information, and it can guarantee the normal tourist traffic through the effective means to ease tourists with information system ${ }^{[2]}$. According to the practice of travel agencies and the $\mathrm{R} \& \mathrm{D}$ requirements of tourism transportation dynamic monitoring system, the dynamic monitoring system tourism transportation, includes four major functional modules: transport facilities, transport planning, transportation scheduling, transport statistics. This paper combined the actual situation of travel transportation, applying statistical information technology to analyzing the statistical work of tourism transportation, thus a reasonable basis of scientific management can be provided for the statistics of tourist traffic and transportation. According to the design principle of the system, research and design planning and management system based on GIS technology, the construction of framework of the system from top to bottom is divided into three layers: application layer, function layer and data layer. The data layer is the most basic component system, is the underlying database system, the main is to store and manage effectively on the basis of the attribute data and spatial data and basis of the planning result data, mainly through the Access, Excel and other database management and its attribute data, and associated with spatial data; function layer according to the management, visualization and analysis and drawing, sharing function needs to design a planning department; application layer top layer is 
mainly for the data statistics and analysis, drawing, application system which has the function of combining basic data layer analysis, get the decision-making, to promote the rational planning of tourism transportation system.

\section{Literature reviews}

The national government did not communicate information efficiently to the regional governments, providing information which seemed to support nuclear safety ${ }^{[3]}$, whilst not clarifying its limitations and not providing information which presented nuclear power as a threat (NAIIC, 2012d and NAIIC, 2012d). In information science, studies of escalation theory have tended to concentrate on information systems failure, researching why people continue to escalate the amount of resources assigned to a project even when the likelihood of success is low (Drummond, 1999, Keil et al., 1994 and Keil et al., 2000). This paper explores the applicability of escalation theory to forms of information failure outside the scope of information systems and it aims to understand if information avoiding behaviors represented an escalation of commitment to maintaining the status quo within the statistical information related to tourism transport ${ }^{[4]}$. Benefits of statistical information, in development and in reuse, are often predicated on identifying appropriate domain ontology, that are readily available to software developers for tourism enterprises. These can then guide tourism transport work product modeling and verification linked to statistical information. Ontology can also facilitate the interoperability of tourism enterprises' products (service) and the continuing operation of a correct system ${ }^{[5]}$. For example, ontologism can be read by future users of a system, and as a joint development element with the tourists, they can be used to validate and improve the quality of tourism management products (service) during various phases of the development process. They can improve the outcome of various requirement engineering activities. For example, they can improve elicitation by bridging common communication gaps between users and developers.

In modern society, the growth in transportation demand with statistical information is much faster than the growth of urban transport systems, as the resources available for expanding information system capacity remain limited. Therefore, it is necessary to plan and design an effective traffic network. It is, however, a well-known paradox that it is possible to make the network performance worse if traveler behaviors are not considered in the network design. Hence, describing choice behaviors is the basis of network design. This study has been carried out from the perspective of the tourism transport, by which it follows that an analysis of tourism information system from the perspective of tourism consumer demand is also necessary for tourism transport, as well as a segmentation of website users to describe the characteristics of the target audience.

\section{System analysis and design}

3.1 The overall function design of the system. Database resource management is the management of tourism traffic system of the society, economy, population, the planning results and other data. It is composed of the placename management, index, resource library, legend and Hawkeye 5 pages. Resource library page is mainly management of tourism traffic economic belt system database resources, to form the association of directory tree of the the database, add the resource nodes related to the tourism transportation system in the system ${ }^{[6]}$. The applications of statistical information have not changed a great deal through ASP's development. Granted, the actual inner workings have definitely changed dramatically, but the application concept itself has not; the application of statistical information is still defined as the developer-created files and directories that can be requested, invoked, and processed through statistical information within its local directory structures. The tourist traffic and transportation management of travel agencies can give full play to statistical information, advice, and supervision role based on the technology of statistical information, and the use of modern means of dealing with travel agencies, statistical information has become to be necessary. A complete statistical information-processing work process should include four stages: the statistical design based on statistical information with 
statistical surveys, statistical collation, statistical analysis and the development and application of statistical data. However, in terns of the current status quo of tourist traffic and transportation management of travel agencies, statistical work is busy with a variety of daily and monthly accounting login work, which not only can not take into account the statistical analysis and the development and application of statistical data, but also can not be convenient and efficient to transform the statistical data to the information of statistical data. As the statistical tools of exploration and innovation are not enough, resulting in the timeliness of statistical information to provide with lagging lack of predictability, affecting the accuracy of decision-making, the main reason is the traditional means of information processing ${ }^{[7]}$. Therefore, to liberate statisticians to be from the complex to the number of operations, more energy should be carried out to develop and use statistical analysis and statistical data to produce high-quality statistical products, and the application of computer technology for processing of statistical information is essential.

In the tourist traffic and transport system for everyday use, we must follow the laws of science on the transmission system maintenance, and strive to eliminate all possible incidents in the bud. According to the actual use of equipment, the environment, and a variety of equipment, technical requirements, a detailed equipment maintenance program can be brought about. In terms of regular cleaning and maintenance measures for the equipment, a list of conditions should be developed for a variety of related equipment running status, time, and maintenance to make the equipment operation and maintenance at a glance. In order to ensure transmission quality, transmission system should be paid attention to the work of transformation. With the rapid development of television technology, a variety of devices continue to emerge, whose technical indicators are along rising. Transmission system should be tested on a regular basis, and the test results should be through a careful analysis, making the maintenance of the old and new systems. System help module can help the users understand the various kinds of information, such as software operating environment and requirements, and introduce the function of the software installation, system function, the actual operation and feedback methods, as well as, the tourist traffic and transportation information inquiry. The module consists of "system to help" and "About ..." two forms composition, and therefore will not repeat them because of simple structure diagram. The use of the role of System help module is administrators and ordinary users generally. Its function may provide equipment management system for the use of help, displaying system and its version number and other information in fact. System help module moves troops the CHM format document under the system path, which introduces the operating environment of this system and various sub-modules detailed instructions to enable new users to quickly operate the system.

3.2 Analysis of system function module. System maintenance module includes some sub-modules, such as system initialization, user management, data maintenance and data encoding. The use of system maintenance module can achieve the system initialization, the administrator logging on, users adding, deleting and other operations, and setting the appropriate operation of their competence. System maintenance module allows keeping a backup of data sent to the hard disk storage. When the data are loss, the hard drive can restore the data after receiving the information. At the same time, allowing a number of important data has been damaged after repair; the use of data encoding sub-module can be achieved stations (airports, terminals), lines and equipment, materials and other information coding for scientific management and statistical summary of relevant information. System help module is very important for practical applications of the tourist traffic and transport system, which is now a standard help document format on the Windows platform, and also a help document format strongly recommended by Object ARX. System help module includes document management, contacts \& communications group and individual message settings. System help module management includes document management, such as Inbox, Outbox, view of fax sent, trash, etc. Contacts and communication group mainly carry on the management for customer contacts and communications group to facilitate fax mass, and to improve work efficiency. The module includes adding contact information, modifying contact information, deleting contact information, newly creating communication group, modifying communication group, deleting the communication group and so on. Personal information setting is 
mainly to modify the personal information, which includes revising the password, changing contact and so on.

3.3 System database design In view of the resources page shows all the Resources Planning Atlas nodes, this resource information can be displayed in the list of resources. In the resource list window, use of keywords or code query to find all kinds of resources in the list in different columns describe various resource information, select a resource can delete, modify the resource information, at the same time in resource view and delete, modify the information resources in the view of resources. Using the tools of ORACLE provided by the Enterprise Manager Console and PLSQR Developer is very convenient to design, develop, deploy and manage the database ${ }^{[8]}$. The use of statistical information for tourism Enterprise Manager Console to design, develop, deploy and manage is of visualization. The statistical input information, statistics query information, tourism and transport sector information, user information, etc in database were stored in different tables. Among them, statistical input information table and so on.

3.4 System Development Environment In the map view page, according to the demand of map finishing, analysis and mapping; spatial distribution using the spatial query to find resources, find the region to highlight the flashing display, display the attribute information area, and to find the regional operation by scaling, can only display the search to regions. Traditional server scripting languages, such as statistical information and other information system, are embedded in the HTML code for the interpretation or implementation of methods to compile a script prepared by the server from the server platform to generate HTML by the implementation of these codes. The application of statistical information thoroughly broken this tradition, whose implementation has to go through translator-editor twice: from scripting language to MSIL (Microsoft Intermediate Language, Microsoft Intermediate Language) and from MSIL to machine code, getting rid of the interpreted language. It uses a structured web pages, through the Code Behind technology to enable separate the logical code and the performance code, by which the two codes do not affect each other. The concept of server-side events is added in, changing the pattern of scripting languages to be closer to Windows programming. Planning analysis drawing module: it is the system of population, economy, society, natural environment, resources and other aspects, including maps, vector, images, pictures, such as grid format data display, analysis, mapping, is the system one of the core module. Mainly includes the map display, query, graphics and other functions. Map operation function mainly to form layers, such as topography, rivers, vegetation, soil and other factors, the system can on the displayed map coding series, finishing, including zoom, roaming, add legend, display scale; query function mainly through the spatial query and Expression Query (combination query, fuzzy query) to find out the attribute data or distribution; mapping function mainly from the repository read data (spatial data, statistical data), by symbol the system call layer element symbol library, which is based on statistical index data and geographical map, by calling the statistical mapping Wizard (histogram, pie chart, fan charts etc.), statistical selection structured symbols and graphics fields, using a different color and the parameters of the thematic mapping.

\section{Model construction}

4.1 Authentication technology. The system utilizes a three-tier security model of user - role module: the first layer is for the user, the second layer for role, and the third layer for module. The relationships between users and roles, roles and the module permissions have been established, while the users and the module permissions have no direct relations. This model separates the system module permissions and users to make the role be as a middle layer. The relationship between users and roles is that a user can simultaneously belong to one or more of the roles and a role can also contain one or more users at the same time. Similarly, the relationship between roles and modules are also the relationship of many to many and the role of the module can be set permissions to specific operations.

All the information in the world of computer networks, including the user's identity information is to use a specific set of data to recognize the user's digital identity for the computer only, and all authorized users of digital identity is authorized for the others. How to ensure the operation of a 
digital identity digital identity operator is the lawful owner of the physical, which means to ensure that the operator corresponding to the identity and digital identity. Identity authentication technology is to solve this problem, and, as the protection of the first pass network assets, it has a pivotal role in authentication.

When the users visit the modules, they can obtain the permissions to visit the module through their role in the access to the module, by which an effective permission management can be achieved $^{[9]}$. In this way, when users enter the module, all operations of the user's must go through permission judgment. When they have no operation permissions authority to determine and do not have permission to operate, the tips are given to prohibit users' access.

In the design of the database, the length of the numeric field, significance and the relationship between data tables should be considered. Part of the data structure is shown in Figure 1.

\section{NETWORKING SOLUTIONS}

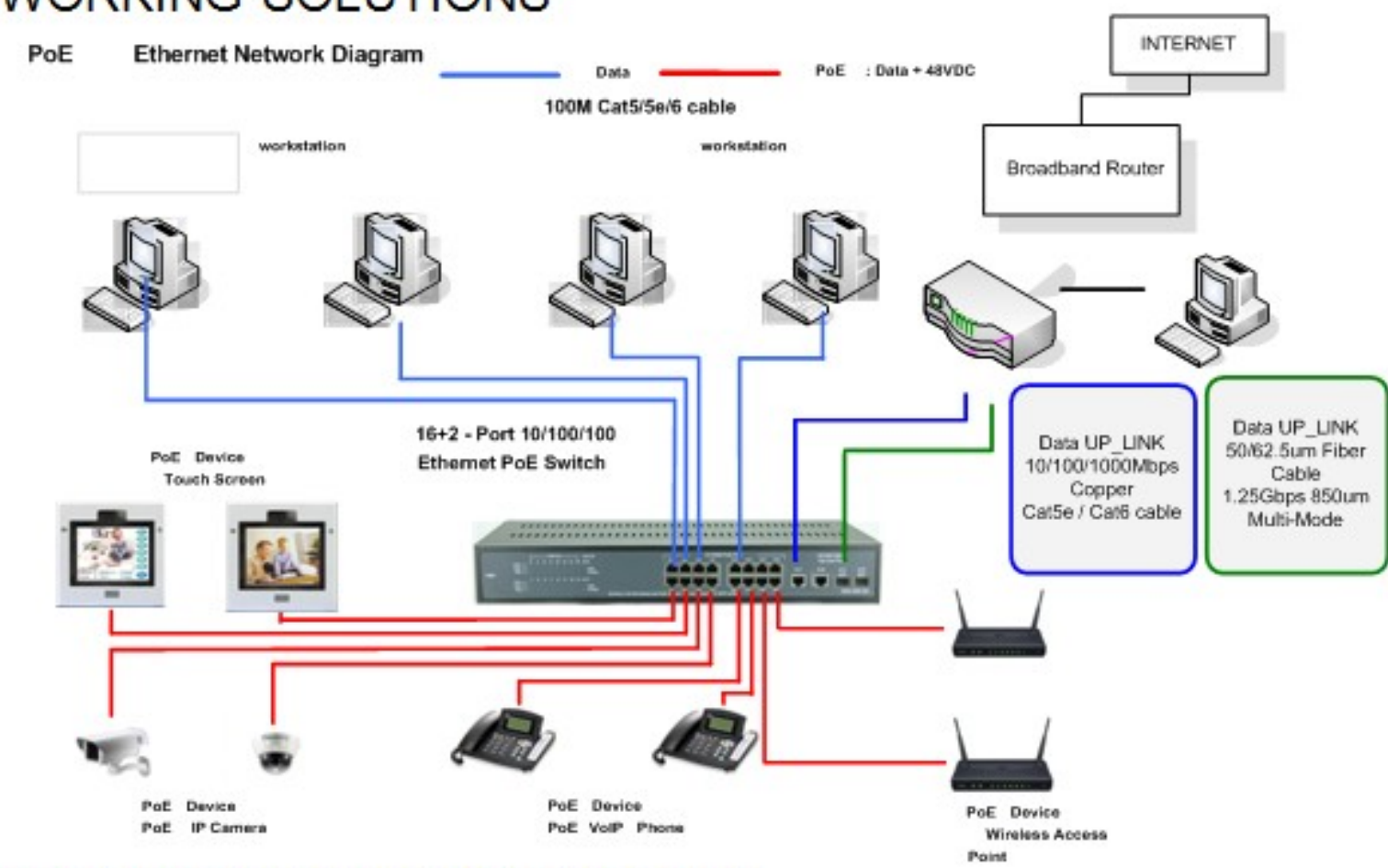

Model: $16+2$ Port Gigabit PoE switch Rev 1.1 09/01/2011 Page 1 of 2

Figure 1 the significance and the relationship between data

4.2 themes and skin technology. The functions of statistical information themes and skin (including the master pages) are not only very easy to achieve a coherent style of the entire site, but also can be very easy to dynamically change the style of the entire site. Fortunately, the better news is that these features provide more consistent with the concept of software engineering, to make the project team collaboration be easier and more conventional in the website development process. The so-called "theme" refers to a collection of the appearance of the page and the appearance property of control settings. The expressed layer is the level of being direct to contact with customers, including the features, interface and so on which can seen directly. Logic layer focused on transaction processing, also known as the middle layer.

\section{Conclusions}

Planning management system of tourism transportation system using GIS technology, the use of C\# language in the Windows environment, can establish of planning and resource database. On the one hand, to strengthen the foundation data management, analysis and mapping analysis and applications, such as mapping, planning analysis, planning achievements management and other functions, on the other hand, realize the sharing, data visualization. The system will improve the technical level of planning and management, provide a good platform for the planning and management departments, the relevant 
departments to promote the reasonable planning of the tourism transportation system and layout, to promote the sustainable development of travel industry, to lay a good foundation for planning. In this paper, by considering the travel time reliability, the statistical information system of tourist traffic and transportation in travel agencies, as one four major functional modules of dynamic monitoring system, played a statistical analysis and support decision-making role. The system can obtain the planning and related information of tourist traffic and transportation from three major functional modules of transportation resources, transportation plan, transportation scheduling. The use of this information, the managers can study transport statistics to reasonably accurately analysis tourist traffic and transportation, which become the key to success for monitoring tourist traffic and transportation of travel agencies and transportation. The statistical system reliability will be affected by other factors such as tourists' demand and variation of travel time perceptions of suppliers.

\section{References}

[1] Abdelzaher et al., 2002 T. Abdelzaher, K.G. Shin and N. Bhatti, Performance guarantees for web server end-systems: a control-theoretical approach, IEEE Transactions on Parallel and Distributed Systems 13 (2002) (1).

[2] Brachman, 2002 R.J. Brachman, Systems that know what they're doing, IEEE Intelligent Systems 17 (2002) (6), pp. 67-71.

[3] Bugnyar and B. Heinrich, Ravens, Corvus corax, differentiate between knowledgeable and ignorant competitors, Proc. R. Soc. Lond. B. Biol. Sci. 272 (2005), pp. 1641-1646.

[4] C.G. Paz-y-Mino, A.B. Bond, A.C. Kamil and R.P. Balda, Pinyon jays use transitive inference to predict social dominance, Nature 430 (2004), pp. 778-781.

[5] Chen et al., 2004 Chen, M., Zheng, A., Lloyd, J., Jordan, M., Brewer, E., 2004. Failure diagnosis using decision trees. In: Proceedings of the International Conference on Autonomic Computing (ICAC-04), pp. 36-43.

[6] Clark et al., 2003 D.D. Clark, C. Partridge, J.C. Ramming and J. Wroclawski, A knowledge plane for the internet, Proceedings of ACM SIGCOMM (2003).

[7] Wilson, 1999 T.D. Wilson. Models in information behaviour research. Journal of Documentation, 55 (3) (1999), pp. 249-270

[8] Wilson, 2000 T.D. Wilson. Human information behavior. Informing Science, 3 (2) (2000), pp. 49-56.

[9] Wong, 2005 K.F.E. Wong. The role of risk in making decisions under escalation situations. Applied Psychology: An International Review, 54 (4) (2005), pp. 584-607. 